\title{
Sangrado del intestino delgado: enfoque y tratamiento
}

\section{Small Bowel Bleeding: Approach and Treatment}

Carmenza Sandoval Riveros¹, Adán Lúquez Mindiola², Hernando Marulanda Fernandez², William Otero Regino³.

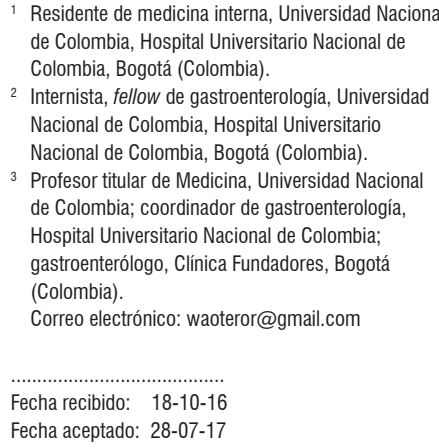

2 Internista, fellow de gastroenterología, Universidad Nacional de Colombia, Hospital Universitario Nacional de Colombia, Bogotá (Colombia).

3 Profesor titular de Medicina, Universidad Nacional de Colombia; coordinador de gastroenterología, Hospital Universitario Nacional de Colombia; gastroenterólogo, Clínica Fundadores, Bogotá (Colombia).

Correo electrónico: waoteror@gmail.com

\begin{abstract}
Resumen
Actualmente, se pueden identificar lesiones del intestino delgado que antes eran inaccesibles. La principal herramienta diagnóstica es la videocápsula endoscópica por el alto valor predictivo negativo. Con los avances en los métodos endoscópicos, la clasificación del sangrado gastrointestinal ha cambiado. Es así como la definición del sangrado oscuro, que antes incluía al originado en el intestino delgado, se ha relegado cuando su origen no se puede identificar tras la realización de una esofagogastroduodenoscopia, colonoscopia y estudios del tracto digestivo medio, tales como videocápsula endoscópica, enteroscopia de empuje, enteroscopia profunda, enteroscopia intraoperatoria, enterorresonancia, enterotomografía, angiografía y gammagrafía.
\end{abstract}

\section{Palabras claves}

Hemorragia de intestino delgado, sangrado gastrointestinal oscuro, videocápsula endoscópica, enteroscopia, enterografía.

\begin{abstract}
Intestinal lesions that were previously inaccessible can now be identified. The most important new diagnostic tool is the endoscopic videocapsule because of its high negative predictive value. With advances in endoscopic methods, the classification of gastrointestinal bleeding has changed so that definition of occult and obscure bleeding that previously included bleeding originating in the small intestine has been relegated to cases for which the origin cannot be identified after performing esophagogastroduodenoscopy, colonoscopy and studies of the middle digestive tract such as endoscopic videocapsule, push enteroscopy, deep enteroscopy , intraoperative enteroscopy, MRI enterography, CT enterography, angiography and scintigraphy.
\end{abstract}

\section{Keywords}

Small intestinal hemorrhage, obscure gastrointestinal bleeding, endoscopic videocapsule, enteroscopy, enterography. 
Se recomienda repetir la EVDA cuando hay hematemesis o melenas recurrentes, y la colonoscopia cuando hay hematoquezia recurrente (4). Con la segunda EVDA, la causa del sangrado se identifica en el $2 \%$ al $25 \%$ de los casos y, con la segunda colonoscopia, en el $6 \%$ al $23 \%$ de ellos (5). Con los métodos actuales que evalúan el ID, se ha demostrado que ese segmento explica entre el $5 \%$ y el $10 \%$ de todos los sangrados gastrointestinales y hasta el $75 \%$ de todos los sangrados anteriormente considerados de origen oscuro (6).

Anatómicamente, el sangrado del ID incluye cualquier sitio comprendido desde la ampolla de Váter hasta la válvula ileocecal. Al sangrado de este segmento también se le ha denominado "sangrado de origen intermedio" (7). El ID tiene entre 6 y $7 \mathrm{~m}$ de longitud y $2,5 \mathrm{~cm}$ de diámetro. Consta del duodeno $(30 \mathrm{~cm})$, el yeyuno $(250 \mathrm{~cm})$ y el íleon $(350 \mathrm{~cm})(8)$. Por su longitud y disposición anatómica, era un órgano difícil de estudiar. Sin embargo, con el advenimiento de nuevas tecnologías, en la actualidad, puede ser evaluado (9). Cuando no se encuentra el origen del sangrado después de examinar todos los segmentos mencionados, el diagnóstico es "sangrado gastrointestinal de origen oscuro" (10), tal y como se resume en la Figura 1.

Los métodos disponibles para estudiar el ID son la enteroscopia de empuje, la enteroscopia asistida por balón, la enteroscopia espiral, la videocápsula endoscópica (VDCE) y la enterografía por tomografía o por resonancia magnética y la enteroscopia intraoperatoria (11). La enteroscopia intraoperatoria se considera la "regla de oro" para estudiar el ID. Otros métodos complementarios para localizar el origen del sangrado son la arteriografía, la angiorresonancia, la angioTAC y la gammagrafía (12).

\section{METODOLOGÍA}

Para la realización del presente trabajo, la búsqueda de la bibliografía se realizó utilizando los siguientes términos $\mathrm{MeSH}$ y palabras clave en la estrategia de búsqueda: small bowel bleeding, gastrointestinal bleeding, obscure bleeding, occult bleeding, overt bleeding, capsule endoscopy, single-balloon enteroscopy, double-balloon enteroscopy, push enteroscopy, spiral enteroscopy, angiography, iron-deficiency anemia, magnetic resonance enterography, Meckel's diverticulum, deep enteroscopy, intraoperative enteroscopy, CT enterography, scintigraphy. La búsqueda se limitó a estudios realizados en humanos, tanto en inglés como en español, y publicados desde el año 2005 hasta octubre de 2016. Las bases de datos electrónicas investigadas fueron Cochrane, Central de Ensayos Controlados, MEDLINE, EMBASE y Science Citation Index. También se realizaron búsquedas manuales. Después de la identificación de las publicaciones, se eligieron las que, en concepto de los autores, eran las más relevantes.

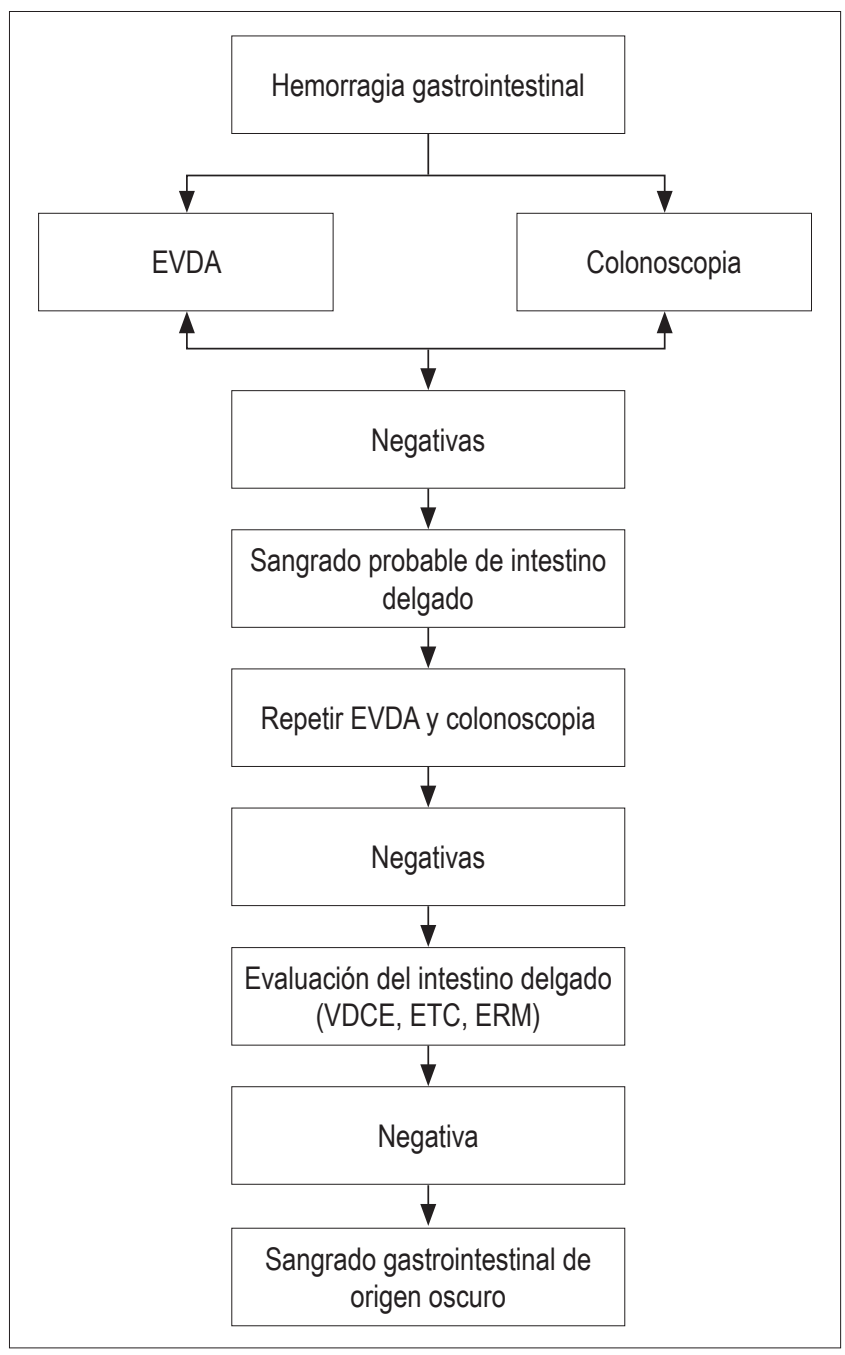

Figura 1. Definición del sangrado probable de intestino delgado y del sangrado de origen oscuro. EVDA: endoscopia de vías altas digestivas; VCE: videocápsula endoscópica; ETC: enteroTAC; ERM: enterorresonancia.

\section{MANIFESTACIONES CLÍNICAS}

Un paciente tiene "sangrado potencial" del ID cuando la EVDA y la CLN son negativas. Clínicamente, puede ser perceptible o manifiesto con melenas o hematoquezia (70\%) o puede ser oculto (30\%). Este último se identifica por anemia ferropénica o sangre oculta positiva en heces (13).

\section{Etiología del sangrado en intestino delgado (SID)}

Las causas de SID son múltiples e incluyen aquellas de origen vascular, inflamatorio y tumoral. Las diferentes causas varían con la edad (Tabla 1) y las zonas geográficas. En Corea del Sur, se ha encontrado como causas más frecuentes las úlceras 
Tabla 1. Etiología del sangrado en intestino delgado.

\begin{tabular}{|c|c|c|c|}
\hline & \multicolumn{2}{|c|}{ Causas comunes $(75 \%)$} & \multirow[t]{2}{*}{ Causas raras } \\
\hline & Menores de 40 años & Mayores de 40 años & \\
\hline \multirow{2}{*}{$\begin{array}{l}\text { Causas } \\
\text { vasculares } \\
(24 \%)\end{array}$} & Angiodisplasia (9\%) & $\begin{array}{l}\text { Angioectasia ( } 54 \% \text { en }>65 \text { años } \\
\text { y 35\% en } 41-64 \text { años) }\end{array}$ & Várices en intestino delgado o enteropatía hipertensiva portal \\
\hline & Lesiones de Dieulafoy & Lesiones de Dieulafoy & $\begin{array}{l}\text { Síndrome del nevus gomoso azul o síndrome de Bean } \\
\text { Síndrome de Osler-Weber R }\end{array}$ \\
\hline \multirow{4}{*}{$\begin{array}{l}\text { Causas } \\
\text { inflamatorias } \\
(18 \%)\end{array}$} & Enfermedad de Crohn (34\%) & Úlceras por AINE: 13\% & \\
\hline & Úlceras intestinales & & \\
\hline & Divertículo de Meckel & & \\
\hline & Enteritis inespecífica (11\%) & Enteritis inespecífica (10\%) & \\
\hline $\begin{array}{l}\text { Tumorales } \\
(11 \%)\end{array}$ & Tumores intestinales (23\%) & $\begin{array}{l}\text { Neoplasias (13\%): TCEGI, } \\
\text { linfoma, carcinoide, } \\
\text { adenocarcinoma }\end{array}$ & Sarcoma de Kaposi \\
\hline \multirow[t]{9}{*}{ Otros } & Síndrome de poliposis & & Vasculitis por $\lg A$ \\
\hline & & & Pseudoxantoma elástico \\
\hline & & & Amiloidosis \\
\hline & & & Síndrome de Plummer-Vinson \\
\hline & & & Síndrome de Ehlers-Danlos \\
\hline & & & Poliposis hereditaria (PAF, PJ) \\
\hline & & & Papulosis atrófica maligna \\
\hline & & & Hematobilia \\
\hline & & & Fístula aortoentérica \\
\hline
\end{tabular}

PAF: poliposis adenomatosa familiar; PJ: Peutz-Jeghers; AINE: antiinflamatorios no esteroideos; TCEGI: tumor de células del estroma gastrointestinal. Tomada de las referencias 1, 2, 3, 10.

(26\%), las angiodisplasias (10\%), las erosiones (8\%) y los tumores del intestino delgado (2\%) (14). En los países occidentales, el $70 \%$ son de origen vascular, y las angioectasias son las más frecuentes (20\%-55\%), seguidas por los tumores (10\%-20\%) y la enfermedad de Crohn (2\%-10\%) (15).

\section{ENFOQUE CLÍNICO}

El abordaje de los pacientes con SID se inicia con una buena historia clínica (anamnesis y examen físico completos). Rara vez el sangrado es severo (16). En esos casos, la prioridad es la estabilización hemodinámica del paciente (17). Clínicamente, se puede manifestar con melenas o hematoquezia, las cuales dependen en primera medida de la magnitud del sangrado y del tránsito intestinal. Por lo tanto, estas formas no son útiles para predecir el origen del sangrado (18). Se deben indagar comorbilidades, como enfermedad de von Willebrand, enfermedad cardíaca valvular, hemodiálisis, hipertensión portal, vasculitis o amiloidosis, uso de aspirina, AINE o anticoagulantes, procedimientos previos (biopsia hepática, trasplante de hígado, reparación de aneurisma aortoabdominal, resección intestinal, radioterapia) y, también, antecedentes familiares de enfermedad inflamatoria intestinal, poliposis, enfermedades malignas y telangiectasia familiar (19). Los adultos mayores con enfermedad cardíaca valvular, enfermedad renal crónica o enfermedad del tejido conectivo tienen alto riesgo de tener lesiones vasculares del ID con riesgo de sangrado. Al examen físico, se deben buscar patologías predisponentes, como telangiectasia hemorrágica hereditaria (telangiectasias en labios u orofaringe), sarcoma de Kaposi (máculas de violáceas a café oscuro en piel y mucosas), síndrome de Peutz-Jeghers (máculas café oscuro en la mucosa de la boca y alrededor de los labios), pseudoxantoma elástico (pápulas amarillentas que pueden confluir formando placas en cuello, codos, fosas poplíteas y región umbilical), síndrome de Ehlers-Danlos (hiperlaxitud y cicatrices papiráceas), síndrome de Bean (nódulos de color azul), vasculitis por IgA (púrpura palpable) o neurofibromatosis (manchas "café con leche" y neurofibromas subcutáneos) (20). 


\section{MÉTODOS DIAGNÓSTICOS}

La tecnología actual ha superado las limitaciones de las pruebas radiológicas tradicionales. Las indicaciones, ventajas y limitaciones de las diferentes modalidades que evalúan el ID se discutirán a continuación.

\section{Videocápsula endoscópica (VCE)}

Se considera el examen de elección para examinar el ID. Es un dispositivo digerible y desechable que mide $26 \times$ $11 \mathrm{~mm}$ y que, generalmente, se expulsa de 8 a 72 horas después (21). Contiene una cámara de video, una fuente de luz, un transmisor de radio y baterías. Toma imágenes a una velocidad de 2 fotografías/segundo durante 8 a 12 horas. Las imágenes se transmiten a una grabadora que se ubica en el abdomen del paciente. Los datos almacenados en esta última y se descargan en un computador que tiene un software específico para su análisis (22). Fue creada en 1981 e introducida en los Estados Unidos en 2001. Evalúa todo el intestino delgado en el 79\% al 90\% de los pacientes. Su rendimiento diagnóstico es del 38\% al 83\%, con una sensibilidad del $95 \%$ y una especificidad del $75 \%$, un valor predictivo positivo del $94 \%$ al $97 \%$ y un valor predictivo negativo del $83 \%$ al $100 \%$ (23).

La probabilidad de encontrar lesiones mediante este método se correlaciona positivamente con las siguientes circunstancias: hemoglobina menor de $10 \mathrm{~g} \%$, duración del sangrado mayor de 6 meses, más de un episodio de hemorragia y sangrado manifiesto frente a oculto (60\% frente a $46 \%$, respectivamente) (24). También hay mayor rendimiento en hombres, en personas mayores de 60 años, en pacientes hospitalizados, en presencia de comorbilidades cardíacas y renales, y en casos en los que el examen se realiza dentro de las 2 semanas del episodio de sangrado (91\% frente a 34\%) (25). Su máximo rendimiento se logra cuando el examen se realiza entre las 48 y las 72 horas del sangrado (26).

Previo al estudio, el paciente debe ser preparado como para una colonoscopia. La recomendación actual es utilizar polietilenglicol (PEG) diluido en 2 litros de agua, bien sea como monoterapia o en combinación con simeticona, para garantizar una mejor calidad en la preparación y, por ende, una mayor rendimiento diagnóstico (27). Luego de dos horas de haber iniciado el estudio, el paciente puede tomar líquidos y, pasadas cinco horas, alimentarse normalmente (28). La cápsula se ingiere sin dificultad en la mayoría de los pacientes. Cuando hay trastornos de la deglución, es necesario colocar la cápsula mediante endoscopia directamente en el estómago o en el intestino delgado. Para su avance con el endoscopio, se necesitan accesorios especiales que incluyen el AdvanCE, asas de polipectomía (para agarrarla) y sobretubos (29).
La única contraindicación formal es la sospecha de obstrucción intestinal debido al riesgo de que la cápsula quede retenida y sea necesaria su extracción quirúrgica (30). Se han considerado contraindicaciones relativas al embarazo, dispositivos de asistencia cardíaca, gastroparesia diabética, demencia y divertículos de Zencker (31).

\section{Ventajas}

Es bien tolerada por los pacientes, no es invasiva, produce molestias mínimas, puede examinar todo el ID y permite decidir la ruta más cercana para llegar a la lesión cuando se necesite realizar una enteroscopia complementaria, bien sea por la boca (anterógrada) o por el ano y avanzar a través de la válvula ileocecal (retrógrada) (32). Dependiendo del tipo de lesión encontrada, se puede tratar con enteroscopia, angiografía o cirugía.

\section{Limitaciones}

Incapacidad para controlar su movimiento a través del tracto gastrointestinal, dificultad para precisar la localización de la lesión, baja especificidad — con 14\% de hallazgos incidentales en voluntarios sanos y falsos negativos en el 10\% al 36\% de los casos (33) - inaccesibilidad al ID cuando hay reconstrucción en Y de Roux y limitada visión de divertículos (34). En la mayoría de los casos, no se logra identificar la papila y hay bajo rendimiento para las lesiones del duodeno y el yeyuno proximal. También tiene eficacia limitada en la detección de tumores submucosos pequeños, con falsos negativos hasta en el 19\% de los casos (35). Hasta el momento, la VCE disponible es una herramienta diagnóstica. Sin embargo, ya existen prototipos que permiten tomar biopsias o liberar materiales hemostáticos (36). Las características de las VCE disponibles se resumen en la Tabla 2.

Tabla 2. Características de las videocápsulas disponibles.

\begin{tabular}{lccc}
\hline & $\begin{array}{c}\text { MicroCam } \\
\text { (Intromedic) }\end{array}$ & $\begin{array}{c}\text { PillCam } \\
\text { (SB3 Given) }\end{array}$ & $\begin{array}{c}\text { EndoCapsule } \\
\text { (Olympus) }\end{array}$ \\
\hline Tamaño $(\mathrm{mm})$ & $11 \times 24$ & $11 \times 26$ & $11 \times 26$ \\
Peso $(\mathrm{g})$ & 3,4 & 3,4 & 3,5 \\
Resolución $(\mathrm{px})$ & $320 \times 320$ & $256 \times 256$ & $512 \times 512$ \\
Fotos por segundo & 3 & 2 & 2 \\
Batería (horas) & 11 & $8-12$ & $8-12$ \\
Ángulo de visión ( ${ }^{\circ}$ ) & 150 & $140 / 156$ & 145 \\
Visión en tiempo real & $\mathrm{Si}$ & $\mathrm{Si}$ & $\mathrm{Sí}$ \\
\hline
\end{tabular}

\section{Complicaciones}

La principal complicación es la retención de la VCE y ocurre en el $0,75 \%$ al 5,8\% de los pacientes (37). En la enfermedad de Crohn, puede ocurrir hasta en el 13\%, debido a estenosis (38). Por lo anterior, se deben investigar síntomas de obstrucción o documentar ausencia de estenosis utilizando de 
manera preliminar la "cápsula de patencia". Se trata de un dispositivo disoluble compuesto por lactosa y bario, diseñado para disolverse después de 30 horas de ser ingerido, tiempo tras el cual queda solo el dispositivo de registro radiopaco, cuyo tamaño residual es de $3 \times 13 \mathrm{~mm}$, lo cual permite su ubicación y tránsito, incluso por zonas estenóticas (39).

Otras complicaciones menos frecuentes son la aspiración traqueobronquial, especialmente en ancianos con trastornos de la deglución. También se han informado casos muy ocasionales de perforación (40).

El análisis de los hallazgos requiere tiempo y concentración por parte del examinador. Los gastroenterólogos experimentados tardan aproximadamente una hora en visualizar las 50000 imágenes (41). Se han desarrollado software para disminuir el tiempo de examen. El primer programa diseñado para optimizar la interpretación de los hallazgos fue el "indicador de rojos", que es un sistema que identifica los sitios con aumento de píxeles en escala de rojos, cuyo objetivo es facilitar la detección de sangrado (42). Aun así, todavía hay una alta tasa de falsos positivos y negativos. Por lo tanto, la recomendación es que esa herramienta se considere simplemente un apoyo y no se confíe totalmente en ella, ya que su valor predictivo negativo no es del 100\% (43).

Otro sistema incorporado es el QuickView, que permite modificar la velocidad del registro, seleccionar imágenes en función del color y crear videos de corta duración. Aunque se han logrado avances en el tiempo de lectura y una mayor detección de lesiones, todavía la tasa de falsos negativos continúa siendo de alrededor del 12\% (44). Las últimas generaciones de VCE, como la CapsoCam, tienen un campo visual de $360^{\circ} \mathrm{y}$ registran 5 fotos por segundo. Con un rendimiento diagnóstico similar a la Pillcam $(84,8 \%$ frente a $81,8 \%)$, la CapsoCam demanda un tiempo de lectura más largo (32,0 minutos frente a 26,2 minutos), pero detecta más lesiones (108 frente a 85 lesiones) (45).

Para superar la dificultad diagnóstica en el duodeno, se están diseñando métodos para dirigir el movimiento de la VCE mediante un imán externo, utilizando una PillCam modificada (46), así como también dispositivos con la posibilidad de obtener biopsias y opciones terapéuticas, con capacidad de liberar fármacos o dispositivos mecánicos (47). Ejemplos de estos son la NEMO (nanocápsula endoscópica con imagen y biopsia óptica) y el VECTOR (cápsula versátil endoscópica para el reconocimiento y tratamiento de tumores gastrointestinales) (48). Ya existen prototipos que tienen agujas y clips de nitinol dentro de una VCE y permiten administrar inyecciones y colocar los clips, lo cual reemplazaría los tradicionales procedimientos invasivos que tienen importantes tasas de morbilidad (49). Una importante limitación de estos modelos es su pequeño tamaño, que implica disponer solo de volúmenes reducidos de medicamentos precargados y, adicionalmente, la imposibilidad de contener más de un dispositivo mecánico para la hemostasia (50).

Otra posibilidad terapéutica radica en poder producir hemostasia local con métodos basados en la generación de calor, utilizando VCE precargadas con óxido de calcio que sería liberado en el sitio de interés (51). Asimismo, se ha estudiado la posibilidad de poder insuflar dispositivos que produzcan compresión mecánica o que sean autoexpandibles al contacto con líquidos gastrointestinales (52).

\section{Enteroscopia de empuje}

Es un enteroscopio que se ha utilizado desde 1980 para evaluar 50 a 100 distales al ligamento de Treitz. Avanza por "tracción y empuje". Su rendimiento es del 3\% al 70\%, especialmente para lesiones vasculares (53), aunque la mayoría de las lesiones diagnosticadas se han encontrado en lugares accesibles mediante endoscopia digestiva alta. La principal desventaja es la dificultad del avance por la formación de asas. Su utilidad es que permite tratar lesiones proximales (54).

\section{Enteroscopia profunda (EP)}

Su objetivo es lograr examinar una mayor longitud del ID como método diagnóstico y terapéutico de estenosis (dilataciones), hemostasia endoscópica (plasma de argón, clips, inyección) y polipectomías. Se realiza con enteroscopios asistidos con balón (uno o dos balones) y con el "enteroscopio en espiral”, y se utiliza tanto la vía anterógrada (introducido por la boca), como la retrógrada (anal) (55).

\section{Enteroscopia de un solo balón (EBS)}

Este equipo se introdujo en 2007. Tiene un solo balón en el extremo distal del sobretubo. Por vía anterógrada, alcanza una profundidad que va desde $133 \mathrm{~cm}$ hasta $256 \mathrm{~cm}$ más allá del ligamento de Treitz y, por vía retrógrada, desde 73 $\mathrm{cm}$ hasta $163 \mathrm{~cm}$ por encima de la válvula ileocecal (56). Su rendimiento diagnóstico varía del $47 \%$ al $74 \%$. La tasa de eventos adversos es del 1\% (57).

\section{Enteroscopia de doble balón (EDB)}

Fue introducido en el 2001. Posee un sobrebretubo y un sistema de balón-bomba con dos balones inflables en sus extremos. Estos balones permiten ir plegando el intestino mediante una serie de ciclos de avance y retirada (58). Debe realizarse bajo anestesia y, en promedio, tarda de 1 a 2 horas. La profundidad de la intubación por vía anterógrada es de 240 a 360 cm más del ligamento de Treitz y, por vía retró- 
grada, de 102 a $140 \mathrm{~cm}$ proximal a la válvula ileocecal (59). Su rendimiento diagnóstico es del $60 \%$ al $80 \%$, y el terapéutico es del $40 \%$ al $73 \%$. El examen de todo el ID se logra en el $16 \%$ al $86 \%$ de los casos (60). En sangrados agudos, ha demostrado que es un procedimiento útil, con una menor tasa de sangrados recurrentes (61). Las complicaciones menores se presentan en el 9,1\% de los pacientes e incluyen distensión o dolor abdominal, odinofagia y náuseas. Las complicaciones mayores se presentan en el $0,72 \%$ al $1 \%$ de los pacientes e incluyen pancreatitis aguda, íleo, sangrado, neumonía aspirativa y perforación (62). Esa última generalmente ocurre con polipectomías de gran tamaño. La mortalidad es rara, con una tasa del 0,05\% (63).

\section{ENTEROSCOPIA EN ESPIRAL (EE)}

Este equipo tiene un sobretubo en espiral que avanza en el ID mediante rotaciones y logra una profundidad promedio de $176 \mathrm{~cm}$ a $250 \mathrm{~cm}$ (64). Cuando se utiliza aisladamente, tiene un rendimiento diagnóstico del $33 \%$ (65). Cuando se utiliza después de una cápsula positiva, el rendimiento es del $57 \%$ (66). Su principal ventaja es la menor duración del examen. Sin embargo, es difícil el abordaje por vía retrógrada, a diferencia de los otros métodos (67). Los efectos adversos son variables. Se ha informado odinofagia en el $12 \%$ de los pacientes, desgarros mucosos en el 27\%, trauma esofágico en el 7\% (2) y perforación en el 0,3\% (68).

\section{Enteroscopia intraoperatoria (EI)}

Con esta técnica, se necesita laparoscopia o laparotomía, con las cuales se puede ayudar el avance del enteroscopio a través del ID. El enteroscopio se puede introducir por vía oral o rectal, o por enterotomía. En general, se recomienda esta última (69). El rendimiento diagnóstico está entre el $58 \%$ y el $88 \%$ (70). Cuando la VCE previa es positiva, el rendimiento es del $87 \%$ (71). La tasa global de resangrado es del 13\% al 60\% después de 19 meses de seguimiento. Las complicaciones ocurren en el $0 \%$ al $52 \%$ e incluyen avulsión de los vasos mesentéricos, íleo prolongado, hematoma, infección y perforación (72). La morbilidad global es del 17\%, y la mortalidad del 5\% - la mayoría de los casos están relacionados con comorbilidades médicas (11\%) y quirúrgicas (22\%) — (73). Es un método invasivo que debe reservarse solo para los pacientes que presenten recurrencia del sangrado y requieran múltiples transfusiones u hospitalizaciones después de una amplia evaluación negativa o en quienes no se pueda realizar la enteroscopia por estenosis o adherencias (74).

\section{NaviAid AB (balón de avance)}

Este método utiliza un colonoscopio estándar con un canal de trabajo de al menos 3,7 mm por el cual se avanza un catéter que posee un balón en la punta. La técnica consiste en avanzar el catéter 30 a $40 \mathrm{~cm}$ distal a la punta del colonoscopio, insuflar el balón, anclarlo en las asas intestinales y avanzar el colonoscopio sobre el catéter (75). El ciclo se repite y, así sucesivamente, se va avanzando de manera rápida y segura en el ID. El catéter que se utiliza tiene una longitud de $3500 \mathrm{~mm}$, y el diámetro del balón es de $40 \mathrm{~mm}$ (76). El dispositivo se puede retirar y sustituir a voluntad del operador, según se necesite tomar biopsias o realizar intervenciones terapéuticas. En estudios preliminares, se encontró que tiene un rendimiento diagnóstico del 44\% (77), aún con menor distancia en profundidad que las técnicas convencionales de enteroscopia. Por vía anterógrada, la profundidad de la inserción es de $158 \mathrm{~cm}$ más allá del píloro, y por vía retrógrada es de $89 \mathrm{~cm}$ proximal a la válvula ileocecal. El tiempo medio para alcanzar las distancias mencionadas es de 15,5 minutos (78). En la Tabla 3 se resumen las características de los equipos para examinar el ID.

\section{Técnicas radiográficas}

Estas incluyen enterotomografía, tomografía computarizada por enteroclísis y enterorresonancia. Cada una de ellas tiene un rendimiento diagnóstico promedio del $45 \%$ (79). La enteroTAC utiliza contraste oral y endovenoso, permite detectar lesiones inflamatorias, neoplasias y anormalidades vasculares con un rendimiento diagnóstico similar a la VCE (80), así como también permite determinar el abordaje más apropiado para la enteroscopia (anterógrado o retrógrado). Es principalmente útil para el diagnóstico de lesiones tumorales y es incluso superior a la VCE, con un rendimiento diagnóstico del 94,1\% frente al $35,3 \%$. La edad menor de 40 años y la hemorragia severa son predictores independientes de un mayor rendimiento diagnóstico de la enteroTAC (81). La enterorresonancia es otra opción en pacientes con contraindicación de VCE. Su evidencia en sangrado de intestino delgado es limitada (82), indicada en aquellos con contraindicación para enteroTAC, y se prefiere en personas jóvenes por una menor exposición a la radiación (83). Estos métodos imagenológicos se pueden considerar antes de la VCE en pacientes con enfermedad inflamatoria intestinal, radioterapia y cirugía de intestino delgado previa o sospecha de estenosis en intestino delgado (84). 
Tabla 3. Desempeños de los diferentes dispositivos de enteroscopia.

\begin{tabular}{lcccc}
\hline & Enteroscopia de doble balón & Enteroscopia de balón único & Enteroscopia en espiral & NaviAid AB \\
\hline Rendimiento $(\%)$ & $40-80$ & $41-65$ & $30-65$ & 44 \\
Anterógrada $(\mathrm{cm})$ & $220-360$ & $133-270$ & $175-250$ & $50-350$ \\
Retrógrada $(\mathrm{cm})$ & $75-183$ & $72-199$ & $\mathrm{NC}$ & $20-150$ \\
Duración $(\mathrm{min})$ & $60-123$ & $52-72$ & $32-52$ & $17-23$ \\
\hline
\end{tabular}

\section{Angiotomografía}

Es una opción si el paciente es intolerante al contraste oral. Ha demostrado ser capaz de detectar tasas de hemorragia lenta de $0,3 \mathrm{~mL} / \mathrm{min}(85)$.

\section{Gammagrafía con glóbulos rojos marcados con tecnecio 99}

Puede detectar sangrado gastrointestinal a una tasa de 0,1$0,4 \mathrm{~mL} / \mathrm{min}$ previo a la angiografía selectiva. En los estudios, ha mostrado baja exactitud para localizar la fuente de sangrado (86).

\section{Gammagrafía con tecnecio $99 \mathrm{~m}+$ pertecnetato}

Es útil para diagnosticar el divertículo de Meckel. Su técnica está basada en la captación de aniones de pertecnetato por la mucosa gástrica ectópica. Tiene una sensibilidad del $64 \%$ al $100 \%$ (87). Se pueden producir resultados falsos positivos en las siguientes situaciones: malformaciones arteriovenosas, lesión inflamatoria, úlceras, obstrucción, intususcepción, quistes de duplicación y mucosa gástrica ectópica (88).

\section{Angiografía}

El objetivo de este examen es la localización topográfica y la embolización terapéutica (89). Para detectar el sitio del sangrado, se necesita que la pérdida de sangre sea de al menos 0,5-1 mL/min (90), y de esto depende su rendimiento diagnóstico, el cual es de entre el 50\% y el $75 \%$ si es activa y de menos del $50 \%$ si es lenta o limitada (91). Las complicaciones potenciales son pseudoaneurisma, trombosis arterial, insuficiencia renal, disección e infarto intestinal. También se pueden presentar infecciones o sangrado en el sitio del catéter, con una incidencia del 10\% de los casos (92). En la Tabla 4, se resumen las tasas de rendimiento diagnóstico de las diferentes técnicas que evalúan el intestino delgado.
Tabla 4. Rendimiento diagnóstico de las técnicas que evalúan el intestino delgado.

\begin{tabular}{lc}
\hline \multicolumn{1}{c}{ Prueba } & Rendimiento (\%) \\
\hline Estudio baritado del intestino delgado & 5 \\
Videocápsula endoscópica & $38-83$ \\
Enteroscopia de empuje & 30 \\
Enteroscopia asistida por dispositivo & $51-80$ \\
Enteroscopia intraoperatoria & $58-88$ \\
Enterografía por tomografía computarizada & 45 \\
\hline
\end{tabular}

\section{ENFOQUE DIAGNÓSTICO}

En el enfoque inicial, el rendimiento diagnóstico de la VCE y la enteroscopia ante un sangrado manifiesto es del $92 \%$, a diferencia del sangrado oculto, que es del 44\% (93). La probabilidad de hacer el diagnóstico con VCE o EP disminuye a medida que pasa el tiempo. Si se estudia el sangrado manifiesto dentro de los 10 a 14 días de la presencia de síntomas, se logra identificar la lesión en el $67 \%$, mientras que, a las 3-4 semanas, se disminuye al 33\% (94). El examen de elección para estudiar un posible sangrado del intestino delgado es la VCE, ya que puede cambiar la estrategia de manejo en el $33 \%$ al $66 \%$ de los pacientes y ha demostrado ser superior a la enteroscopia de empuje (rendimiento del 63\% frente al 23\%) (95). También ha reducido el número de hospitalizaciones, investigaciones adicionales y necesidad de transfusión de sangre y aumentado el rendimiento diagnóstico (73\%-93\%) y terapéutico (57\%$73 \%$ ) de la enteroscopia (96). Por su alto valor predictivo negativo, permite evitar la enteroscopia en pacientes con una baja probabilidad de hallazgos positivos en intestino delgado previa al examen. Si los resultados son negativos y el estado clínico del paciente es estable, se puede hacer un seguimiento sin pruebas adicionales dada la baja tasa de resangrado (97). Sin embargo, para los pacientes con resultados negativos que están tomando anticoagulantes, se requiere una estrecha observación y se deben considerar otras modalidades como alternativa, pero aún no hay indi- 
caciones claras acerca de qué técnica usar ni de cuál es el momento apropiado.

Se recomienda repetir la VCE en los pacientes con disminución de la hemoglobina de al menos $4 \mathrm{~g} / \mathrm{dL}$ o sangrado que pasó de oculto a manifiesto, porque se ha reportado aumento del rendimiento diagnóstico (98), aunque otra opción es realizar la enteroscopia de doble balón que puede detectar la fuente de la hemorragia en el $30 \%$ en este grupo de pacientes. Para seleccionar el tipo de enteroscopia profunda, esto depende de la disponibilidad y la pericia en el manejo de la técnica. En un ensayo multicéntrico prospectivo que comparó ESB y EDB, se demostró una tasa de enteroscopia total 3 veces mayor con EDB en comparación con ESB ( $66 \%$ frente a $22 \%, \mathrm{p}<0,0001)$ y un rendimiento terapéutico no estadísticamente significativo debido al bajo número de participantes ( $72 \%$ frente al $48 \%, \mathrm{p}<0,025$ ) (99). Una revisión comparó el rendimiento diagnóstico de la enteroscopia con un balón, doble balón y en espiral, y encontró resultados similares (53,9\%, 64,4\% y $47 \%$, respectivamente). En cuanto a la duración del procedimiento, el más rápido fue la $\mathrm{EE}$ (oral: 41,0 min., anal: $46 \mathrm{~min}$.) seguida de la ESB (oral: 59,8 min., anal: 68,8 min.) y la EDB (oral: 71,6 min., anal: 84,5 min.).

Las intervenciones terapéuticas fueron mayores en la EDB (40,1\%), en comparación con la ESB y la EE $(26,8 \%$ y $29,7 \%$ ) (100). La diferencia reportada entre la EDB y la EE ha sido el tiempo de inserción, el cual es menor en la EE (43 minutos frente a 65 minutos, $p=0,007$ ), pero la EDB tiene una longitud de alcance mucho mayor $(310 \mathrm{~cm}$ frente a $250 \mathrm{~cm}, \mathrm{p}=0,004)$. El tiempo medio de procedimiento para el enfoque anterógrado se ha estimado en 79 \pm 15 minutos para la EDB, $65 \pm 16$ minutos para la ESB y $35 \pm 6$ minutos para la EE (101). Los metaanálisis que han comparado las diferentes pruebas para el estudio del intestino delgado han presentado variabilidad, con limitaciones por la heterogeneidad y los intervalos de confianza amplios, que disminuyen el nivel de evidencia (102).

\section{SANGRADO MASIVO}

En pacientes hemodinámicamente inestables, se puede considerar realizar de forma urgente una angiografía con-

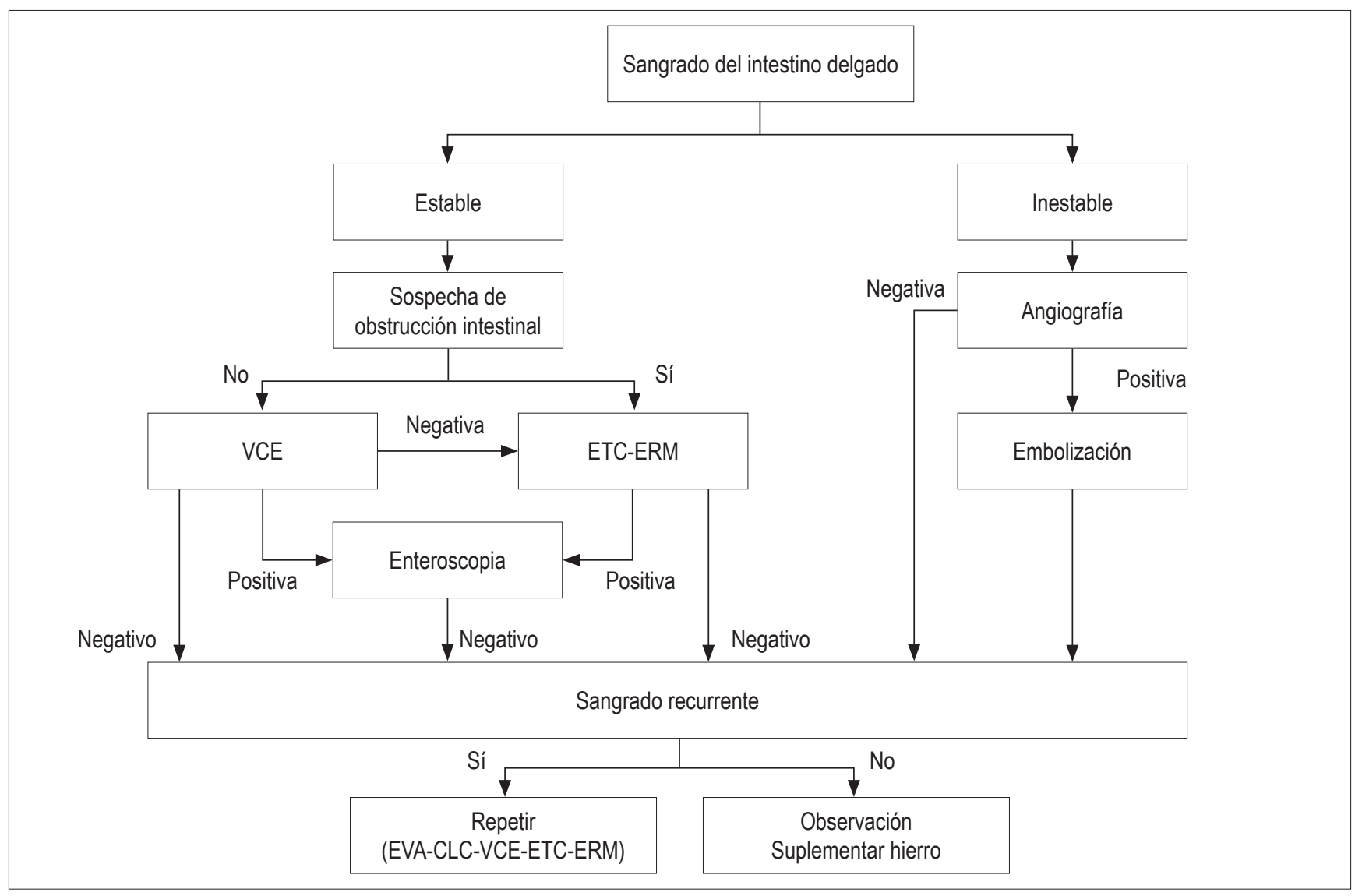

Figura 2. Algoritmo de abordaje del sangrado potencial del intestino delgado. EVDA: endoscopia de vías digestivas altas; CLC: colonoscopia; VCE: videocápsula endoscópica; ETC: enteroTAC; ERM: enterorresonancia. 
vencional o una enteroscopia profunda por la posibilidad de intervención terapéutica. Si el paciente tiene sangrado activo, pero está hemodinámicamente estable, se puede realizar una enterotomografía o angiotomografía para identificar el sitio de sangrado y orientar el manejo (103). En pacientes jóvenes con hemorragia manifiesta, se debe realizar una gammagrafía con tecnecio 99 + pertecnetato para descartar divertículo de Meckel. En los pacientes con hemorragia digestiva manifiesta y tasas más lentas de sangrado $(0,1-0,2 \mathrm{~mL} / \mathrm{min})$, se puede realizar una gammagrafía con glóbulos rojos marcados si la enteroscopia profunda o la VCE no se pueden llevar a cabo (104). En la Figura 2, se esquematiza el abordaje del sangrado del intestino delgado.

\section{EVOLUCIÓN CLÍNICA Y RESANGRADO}

La tasa de resangrado varía en las diferentes publicaciones. La inconsistencia está relacionada con la institución, la duración del seguimiento y la causa de la hemorragia. Son riesgos independientes de resangrado las siguientes alteraciones: transfusiones múltiples y comorbilidades, como insuficiencia renal crónica, uso de anticoagulación y diabetes mellitus (105). Se ha estudiado la tasa de resangrado después de la intervención para las lesiones detectadas en la VCE y encontró que fue del $50 \%$ en pacientes con angiodisplasia a pesar de intervención endoscópica; asimismo, fue mayor para los pacientes con lesiones sin relevancia clínica, independientemente de que se realizara un procedimiento endoscópico (106). En las intervenciones dirigidas por los hallazgos de la VCE, entre el 50\% y el 66\% de los pacientes permanecen sin transfusión y sin sangrado recurrente (84, $85)$, es decir, el riesgo de resangrado después de la VCE negativa es muy bajo, de entre el 5,6\% y el 11\% (107).

Tras 12 meses de una enteroscopia profunda, la recurrencia de sangrado manifiesto ocurrió en el 34\% de los pacientes, en comparación con el $13 \%$ de los pacientes con sangrado oculto $(p=0,06)(87)$. Estas tasas de recurrencia, sin embargo, no fueron significativas a los 30 meses de seguimiento (27\% frente a 20\%) (87). Con la EDB negativa, la tasa es del $30 \%$ al $40 \%$ (42) y, con la EE negativa, del $26 \%$ tras 2 años de seguimiento (108).

\section{CONCLUSIONES}

Recientemente, se han redefinido los conceptos referentes a hemorragias gastrointestinales y se ha introducido uno nuevo, "el sangrado del intestino delgado", el cual explica un alto porcentaje de los clasificados previamente como "de origen oscuro". Esto, en gran parte, es gracias a la posibilidad de detectar lesiones en sitios previamente inaccesibles, lo cual modifica el enfoque clínico y terapéutico. La videocápsula endoscópica continúa siendo la principal herramienta diagnóstica y ofrece opciones terapéuticas con prototipos en desarrollo, además de orientar el abordaje de intervenciones subsecuentes. Las técnicas de enteroscopia ofrecen un rendimiento diagnóstico similar, deben ser elegidas según la disponibilidad y la experiencia del centro. Dentro de las técnicas radiológicas, el enteroTAC es el preferido por sus características, pero, de existir alguna contraindicación, hay múltiples posibilidades. La angiografía es de primera elección en pacientes clínicamente inestables, ya que ofrece una intervención terapéutica rápida. No hay dudas de la gran importancia que revela el tema y del interés que ha despertado a nivel mundial, con un desarrollo exponencial de diversas técnicas que incrementen el rendimiento diagnóstico y permitan disponer de tratamientos que representen una menor morbilidad relacionada.

\section{Declaración de conflicto de interés}

Ninguno declarado por los autores.

\section{Financiación}

Ninguna declarada por los autores.

\section{Agradecimientos}

Ninguno declarado por los autores.

\section{REFERENCIAS}

1. Pasha SF, Hara AK, Leighton JA. Diagnostic evaluation and management of obscure gastrointestinal bleeding: a changing paradigm. Gastroenterol Hepatol. 2009;5(12):839-50.

2. Gerson LB, Fidler JL, Cave DR, et al. ACG Clinical guideline: diagnosis and management of small bowel bleeding. Am J Gastroenterol 2015; 110(9):1265-87. Doi: https:// doi.org/10.1038/ajg.2015.246

3. Ohmiya N, Nakagawa Y, Nagasaka M, et, al. Obscure gastrointestinal bleeding: diagnosis and treatment. Dig Endosc. 2015;27(3):285-94. Doi: https://doi.org/10.1111/ den. 12423

4. Dulic-Lakovic E, Dulic M, Hubner D, et al. Bleeding Dieulafoy lesions of the small bowel: a systematic study on the epidemiology and efficacy of enteroscopic treatment. Gastrointest Endosc. 2011;74(3):573-580. Doi: https:// doi.org/10.1016/j.gie.2011.05.027

5. Min Naut ER. The approach to occult gastrointestinal bleed. Med Clin North Am. 2016;100(5):1047-56. Doi: https:// doi.org/10.1016/j.mcna.2016.04.013

6. Kaufman D, Leslie G, Marya N, et al. Small Intestinal angioectasia: characterization, risk factors, and rebleeding. J Clin Gastroenterol. 2016. Doi: https://doi.org/10.1097/ MCG.0000000000000663 
7. Limsrivilai J, Srisajjakul S, Pongprasobchai S, et al. A prospective blinded comparison of video capsule endoscopy versus computed tomography enterography in potential small bowel bleeding: clinical utility of computed tomography enterography. J Clin Gastroenterol. 2016.

8. Yatagai N, Ueyama H, Shibuya T, et al. Obscure gastrointestinal bleeding caused by small intestinal lipoma: a case report J Med Case Rep. 2016;10(1):226.

9. Liu K, Kaffes AJ. Review article: the diagnosis and investigation of obscure gastrointestinal bleeding. Aliment Pharmacol Ther. 2011;34(4):416-23. Doi: https://doi. org/10.1111/j.1365-2036.2011.04744.x

10. RondonottiE,PennazioM,TothE,etal.Smallbowelneoplasms in patients undergoing video capsule endoscopy: a multicenter European study. Endoscopy. 2008;40(6):488-95. Doi: https://doi.org/10.1055/s-2007-995783

11. Pasha SF, Sharma VK, Carey EJ, et al. Utility of video capsule endoscopy in the detection of small bowel tumors. A single center experience of 1000 consecutive patients. Proceedings of the 6th International Conference on Capsule Endoscopy. June 8-10; Madrid, Spain, 2007. Nueva York: McGraw-Hill; 2007.p. 45.

12. Goenka M.K, Majumder S, Goenka U. Capsule endoscopy: present status and future expectation. World J Gastroenterol 2014;20(29):10024-37. Doi: https://doi.org/10.3748/ wjg.v20.i29.10024

13. Santhakumar C, Liu K. Evaluation and outcomes of patients with obscure gastrointestinal bleeding. World J Gastrointest Pathophysiol. 2014;5(4):479-86. Doi: https://doi. org/10.4291/wjgp.v5.i4.479

14. Upchurch BR, Vargo JJ. Small bowel enteroscopy. Rev Gastroenterol Disord. 2008;8(3):169-77.

15. Lewis BS. Small intestinal bleeding. Gastroenterol Clin North Am. 2000;29(1):67-95. Doi: https://doi.org/10.1016/S08898553(05)70108-4

16. Singh A, Baptista V, Stoicov C, et al. Evaluation of small bowel bleeding. Curr Opin Gastroenterol. 2013;29(2):119-24. Doi: https://doi.org/10.1097/MOG.0b013e32835bdc1a

17. Pennazio M, Spada C, Eliakim R, et al. Small-bowel capsule endoscopy and device-assisted enteroscopy for diagnosis and treatment of small bowel disorders: European Society of Gastrointestinal Endoscopy (ESGE) Clinical Guideline. Endoscopy. 2015;47(4):352-76. Doi: https://doi.org/10.1055/s-0034-1391855

18. Rondonotti E, Villa F, Mulder CJ, et al. Small bowel capsule endoscopy in 2007: indications, risks and limitations. World J Gastroenterol. 2007;13(46):6140-9. Doi: https://doi.org/10.3748/wjg.13.6140

19. Mishkin DS, Chuttani R, Croffie J, et al. ASGE Technology Status Evaluation Report: wireless capsule endoscopy. Gastrointest Endosc. 2006;63(4):539-45. Doi: https://doi. org/10.1016/j.gie.2006.01.014

20. Carey EJ, Leighton JA, Heigh RI, et al. A single-center experience of 260 consecutive patients undergoing capsule endoscopy for obscure gastrointestinal bleeding. Am J Gastroenterol. 2007;102(1):89-95. Doi: https://doi. org/10.1111/j.1572-0241.2006.00941.x
21. Singh A, Marshall C, Chaudhuri B, et al. Timing of video capsule endoscopy relative to overt obscure GI bleeding: implications from a retrospective study. Gastrointest Endosc. 2013;77(5):7616. Doi: https://doi.org/10.1016/j.gie.2012.11.041

22. Yamada A, Watabe H, Kobayashi Y, et al. Timing of capsule endoscopy influences the diagnosis and outcome in obscureovert gastrointestinal bleeding. Hepatogastroenterology. 2012;59(115):676-9.

23. Rocchi A, Benchimol EI, Bernstein CN, et al. Inflammatory bowel disease: a Canadian burden of illness review. Can J Gastroenterol. 2012;26(11):811-7. Doi: https://doi. org/10.1155/2012/984575

24. Lai LH, Wong GL, Chow DK, et al. Long-term follow-up of patients with obscure gastrointestinal bleeding after negative capsule endoscopy. Am J Gastroenterol. 2006;101(6): 12248. Doi: https://doi.org/10.1111/j.1572-0241.2006.00565.x

25. Savale L, Soussan B, Ramírez S, et al. Outcome of patients with obscure gastrointestinal bleeding after negative capsule endoscopy: results of a one-year follow-up study. Gastroenterol Clin Biol. 2010;34(11):606-11. Doi: https:// doi.org/10.1016/j.gcb.2010.06.009

26. Iwamoto J, Mizokami Y, Shimokobe K, et al. The clinical outcome of capsule endoscopy in patients with obscure gastrointestinal bleeding. Hepatogastroenterology. 2011;58(106):301-5.

27. Koh SJ, Im JP, Kim JW, et al. Long-term outcome in patients with obscure gastrointestinal bleeding after negative capsule endoscopy.WorldJGastroenterol.2013;19(10):1632-8.Doi: https://doi.org/10.3748/wjg.v19.i10.1632

28. Kim JB, Ye BD, Song Y, et al. Frequency of rebleeding events in obscuregastrointestinalbleedingwithnegativecapsuleendoscopy. J Gastroenterol Hepatol. 2013;28(5):834-40. Doi: https://doi.org/10.1111/jgh.12145

29. Keller J, Fibbe C, Volke F, et al. Inspection of the human stomach using remote-controlled capsule endoscopy: a feasibility study in healthy volunteers (with videos). Gastrointest Endosc. 2011;73:22-8. Doi: https://doi.org/10.1016/j.gie.2010.08.053

30. Goldstein JL, Eisen GM, Lewis B, et al. Video capsule endoscopy to prospectively assess small bowel injury with celecoxib, naproxen plus omeprazole and placebo. Clin Gastroenterol Hepatol. 2005;3(2):133-41. Doi: https:// doi.org/10.1016/S1542-3565(04)00619-6

31. Lewis BS, Eisen GM, Friedman S. A pooled analysis to evaluate results of capsule endoscopy trials. Endoscopy. 2005;37(10):9605. Doi: https://doi.org/10.1055/s-2005-870353

32. Appleyard M, Fireman Z, Glukhovsky A, et al. A randomized trial comparing wireless capsule endoscopy with push enteroscopy for the detection of small-bowel lesions. Gastroenterology. 2000;119(6):1431-8. Doi: https://doi. org/10.1053/gast.2000.20844

33. Clarke JO, Giday SA, Magno P, et al. How good is capsule endoscopy for detection of periampullary lesions? Results of a tertiary-referral center. Gastrointest Endosc. 2008;68(2):267-72. Doi: https://doi.org/10.1016/j. gie.2007.11.055 
34. Pennazio M, Rondonotti E, de Franchis R. Capsule endoscopy in neoplastic diseases. World J Gastroenterol. 2008;14(34):5245-53. Doi: https://doi.org/10.3748/ wjg. 14.5245

35. NadlerM,EliakimR.Therole of capsuleendoscopyinacutegastrointestinal bleeding. Therap Adv Gastroenterol. 2014;7:8792. Doi: https://doi.org/10.1177/1756283X13504727

36. Van de Bruaene C, De Looze D, Hindryckx P. Small bowel capsule endoscopy: where are we after almost 15 years of use? World J Gastrointest Endosc. 2015;7:13-36. Doi: https://doi.org/10.4253/wjge.v7.i1.13

37. Koulaouzidis A, Karargyris A, et al. Utility of 3-dimensional image reconstruction in the diagnosis of small-bowel masses in capsule endoscopy (with video). Gastrointest Endosc. 2014;80(4):642-51. Doi: https://doi.org/10.1016/j. gie.2014.04.057

38. Pioche M, Vanbiervliet G, Jacob P, et al. Prospective randomized comparison between axial- and lateral-viewing capsule endoscopy systems in patients with obscure digestive bleeding. Endoscopy. 2014;46:479-84.

39. Swain P, Toor A, Volke F, et al. Remote magnetic manipulation of a wireless capsule endoscope in the esophagus and stomach of humans (with videos). Gastrointest Endosc. 2010;71(7):12903. Doi: https://doi.org/10.1016/j.gie.2010.01.064

40. Rey JF, Ogata $\mathrm{H}$, Hosoe $\mathrm{N}$, et al. Blinded nonrandomized comparative study of gastric examination with a magnetically guided capsule endoscope and standard videoendoscope. Gastrointest Endosc. 2012;75(2):373-81. Doi: https://doi. org/10.1016/j.gie.2011.09.030

41. Ortora G, Valdastri P, Susilo E, et al. Propeller-based wireless device for active capsular endoscopy in the gastric district. Minim Invasive Ther Allied Technol. 2009;18(5):280-90. Doi: https://doi.org/10.1080/13645700903201167

42. Yim S, Gultepe E, Gracias DH, et al. Biopsy using a magnetic capsule endoscope carrying, releasing, and retrieving untethered microgrippers. IEEE Trans Biomed Eng. 2014;61(2):51321. Doi: https://doi.org/10.1109/TBME.2013.2283369

43. Muñoz F, Alici G, Li W. A review of drug delivery systems for capsule endoscopy. Adv Drug Deliv Rev. 2014;71:77-85. Doi: https://doi.org/10.1016/j.addr.2013.12.007

44. Ryan Scott. Advances in capsule endoscopy gastroenterology \& hepatology. Gastroenterol Hepatol. 2015;11:612-27.

45. Ross A, Mehdizadeh S, Tokar J, et al. Double balloon enteroscopy detects small bowel mass lesions missed by capsule endoscopy. Dig Dis Sci. 2008;53(8):2140-3. Doi: https:// doi.org/10.1007/s10620-007-0110-0

46. Schostek S, Schurr MO. European research on wireless endoscopy the VECTOR project. Stud Health Technol Inform. 2013;189:193-9.

47. Hale MF, Sidhu R, McAlindon ME. Capsule endoscopy: current practice and future directions. World J Gastroenterol. 2014;20(24):7752-9. Doi: https://doi.org/10.3748/wjg. v20.i24.7752

48. Valdastri P, Quaglia C, Susilo E, et al. Wireless therapeutic endoscopic capsule: in vivo experiment. Endoscopy. 2008;40(12):97982. Doi: https://doi.org/10.1055/s-0028-1103424
49. Pennazio M. Capsule endoscopy: where are we after 6 years of clinical use? Dig Liver Dis 2006;38(12):867-78. Doi: https://doi.org/10.1016/j.dld.2006.09.007

50. Fry LC, De Petris G, Swain JM, et al. Impaction and fracture of a video capsule in the small bowel requiring laparotomy for removalofthecapsulefragments.Endoscopy.2005;37(7):6746. Doi: https://doi.org/10.1055/s-2005-870252

51. Cheifetz AS, Kornbluth AA, Legnani P, et al. The risk of retention of the capsule endoscope in patients with known or suspected Crohn's disease. Am J Gastroenterol. 2006;101(10):2218-22. Doi: https://doi.org/10.1111/ j.1572-0241.2006.00761.x

52. Health Quality Ontario. Capsule endoscopy in the assessment of obscure gastrointestinal bleeding: an evidence-based analysis. Ont Health Technol Assess Ser. 2015;15(1):1-55.

53. Bhattarai $M$, Bansal $P$, Khan Y. Longest duration of retention of video capsule: a case report and literature review. World J Gastrointest Endosc. 2013;5(7):352-5. Doi: https://doi. org/10.4253/wjge.v5.i7.352

54. Repici A, Barbon V, De Angelis C, et al. Acute small-bowel perforation secondary to capsule endoscopy. Gastrointest Endosc. 2008;67(1):180-3. Doi: https://doi.org/10.1016/j. gie.2007.05.044

55. Lin S, Branch MS, Shetzline M. The importance of indication in the diagnostic value of push enteroscopy. Endoscopy. 2003;35(4):31521.Doi: https://doi.org/10.1055/s-2003-38144

56. Raju GS, Gerson L, Das A, et al. American Gastroenterological Association (AGA) Institute technical review on obscure gastrointestinal bleeding. Gastroenterology. 2007;133(5):1697717. Doi: https://doi.org/10.1053/j.gastro.2007.06.008 / https://doi.org/10.1053/j.gastro.2007.06.007

57. LinderJ,CheruvattathR, Truss C, etal.Diagnosticyieldandclinical implications of push enteroscopy: results from a nonspecialized center.J Clin Gastroenterol.2002;35(5):383-6. Doi: https://doi.org/10.1097/00004836-200211000-00005

58. Sidhu R, McAlindon ME, Kapur K, et al. Push enteroscopy in the era of capsule endoscopy. J Clin Gastroenterol. 2008;42(1):54-8. Doi: https://doi.org/10.1097/01.mcg.0000225655.85060.74

59. Sidhu R, Sanders DS. Double-balloon enteroscopy in the elderlywithobscuregastrointestinalbleeding: safetyandfeasibility.EurJGastroenterolHepatol.2013;25(10):1230-4.Doi: https://doi.org/10.1097/MEG.0b013e3283630f1b

60. Baptista V,Marya N, Singh A, etal. Continuing challenges in the diagnosisandmanagementofobscuregastrointestinalbleeding. World J Gastrointest Pathophysiol. 2014;5(4):523-33. Doi: https://doi.org/10.4291/wjgp.v5.i4.523

61. Mönkemüller K, Neumann H, Meyer F, et al. A retrospective analysis of emergency double-balloon enteroscopy for small-bowel bleeding. Endoscopy. 2009;41(8):715-7. Doi: https://doi.org/10.1055/s-0029-1214974

62. Aniwan S, Viriyautsahakul V, Rerknimitr R, et al. Urgent double balloon endoscopy provides higher yields than nonurgent double balloon endoscopy in overt obscure gastrointestinal bleeding. Endosc Int Open. 2014;2(2):E90-5. Doi: https://doi.org/10.1055/s-0034-1365543 
63. Xin L, Liao Z, Jiang YP, et al. Indications, detectability, positive findings, total enteroscopy, and complications of diagnostic double-balloon endoscopy: a systematic review of data over the first decade of use. Gastrointest Endosc. 2011;74(3):563-70.Doi:https://doi.org/10.1016/j. gie.2011.03.1239

64. Moschler O, May AD, Muller MK, et al. Complications in double-balloon enteroscopy: results of the German DBE register. Z Gastroenterol. 2008;46(3):266-70.

65. Khashab MA, Pasha SF, Muthusamy VR, et al. ASGE: the role of deep enteroscopy in the management of small-bowel Disorders. Gastrointest Endosc. 2015;82(4):600-7. Doi: https: / doi.org/10.1016/j.gie.2015.06.046

66. Buscaglia JM, Richards R, Wilkinson MN, et al. Diagnostic yield of spiral enteroscopy when performed for the evaluation of abnormal capsule endoscopy findings. J Clin Gastroenterol. 2011;45(4):342-6. Doi: https://doi. org/10.1097/MCG.0b013e3181eeb74b

67. Schembre DB, Ross AS. Spiral enteroscopy: a new twist on over tuve assisted endoscopy. Gastrointest Endosc. 2009;69(2):3336. Doi: https://doi.org/10.1016/j.gie.2008.09.011

68. Douard R, Wind P, Berger A, et al. Role of intraoperative enteroscopy in the management of obscure gastointestinal bleeding at the time of video-capsule endoscopy. Am J Surg. 2009;198(1):6-11. Doi: https://doi.org/10.1016/j.amjsurg.2008.06.036

69. Bonnet S, Douard R, Malamut G, et al. Intraoperative enteroscopy in the management of obscure gastrointestinal bleeding. Dig Liver Dis. 2013;45(4):277-84.

70. Murino A, Vlachou E, Fraser C, et al. Deep enteroscopy using a conventional colonoscope and through-the-scope balloon catheter system: How deep is deep? Gastrointest Endosc. 2016;84(5):8823. Doi: https://doi.org/10.1016/j.gie.2016.01.031

71. Neumann H. Through-the-scope deep enteroscopy (TTS-DE): new technique for deep small-bowel endoscopy - a pilot study. Z Gastroenterol. 2013;51:K259. Doi: https://doi.org/10.1055/s-0033-1352899

72. Deep enteroscopy with a conventional colonoscope: initial multicenter study by using a through-the-scope balloon catheter system. Gastrointest Endosc. 2015.

73. Howarth DM. The role of nuclear medicine in the detection of acute gastrointestinal bleeding. Semin Nucl Med. 2006;36(2):133-46. Doi: https://doi.org/10.1053/j.semnuclmed.2005.11.001

74. Lee SS, Oh TS, Kim HJ, et al. Obscure gastrointestinal bleeding: diagnostic performance of multidetector CT enterography. Radiology. 2011;259(3):739-48. Doi: https://doi. org/10.1148/radiol.11101936

75. Filippone A, Cianci R, Milano A, et al. Obscure gastrointestinal bleeding and small bowel pathology: comparison between wireless capsule endoscopy and multidetector-row CT enteroclysis. Abdom Imaging. 2008;33(4):398-406. Doi: https://doi.org/10.1007/s00261-007-9271-8

76. Heo HM, Park CH, Lim JS, et al. The role of capsule endoscopy after negative CT enterography in patients with obscure gastrointestinal bleeding. Eur Radiol. 2012;22(6):1159-66. Doi: https://doi.org/10.1007/s00330-011-2374-1

77. Browder W, Cerise EJ, Litwin MS. Impact of emergency angiography in massive lower gastrointestinal bleeding. Ann Surg. 1986;204(5):530-6. Doi: https://doi. org/10.1097/00000658-198611000-00004

78. Leung WK, Ho SS, Suen BY, et al. Capsule endoscopy or angiography in patients with acute overt obscure gastrointestinal bleeding: a prospective randomized study with longterm follow-up. Am J Gastroenterol. 2012;107(9):1370-6. Doi: https://doi.org/10.1038/ajg.2012.212

79. Rahn NH 3rd, Tishler JM, Han SY, et al. Diagnostic and interventional angiography in acute gastrointestinal hemorrhage. Radiology. 1982;143(2):361-6. Doi: https://doi. org/10.1148/radiology.143.2.6978500

80. Silverstein FE, Gilbert DA, Tedesco FJ, et al. The national ASGE survey on upper gastrointestinal bleeding. I. Study design and baseline data. Gastrointest Endosc. 1981;27(2):73-9. Doi: https://doi.org/10.1016/S00165107(81)73155-9

81. Strate LL. Lower GI bleeding: epidemiology and diagnosis. Gastroenterol Clin North Am. 2005;34(4):643-64. Doi: https://doi.org/10.1016/j.gtc.2005.08.007

82. Leaper M, Johnston MJ, Barclay M, et al. Reasons for failure to diagnose colorectal carcinoma at colonoscopy. Endoscopy. 2004;36(6):499-503. Doi: https://doi. org/10.1055/s-2004-814399

83. Descamps C, Schmit A, Van Gossum A. "Missed” upper gastrointestinal tract lesions may explain "occult" bleeding. Endoscopy. 1999;31(6):452-5. Doi: https://doi. org/10.1055/s-1999-151

84. Chak A, Koehler MK, Sundaram SN, et al. Diagnostic and therapeutic impact of push enteroscopy: analysis of factors associated with positive findings. Gastrointest Endosc. 1998;47(1):18-22. Doi: https://doi.org/10.1016/S00165107(98)70293-7

85. Pennazio M, Santucci R, Rondonotti E, et al. Outcome of patients with obscure gastrointestinal bleeding after capsule endoscopy: report of 100 consecutive cases. Gastroenterology. 2004;126(3):643-53. Doi: https://doi. org/10.1053/j.gastro.2003.11.057

86. Arakawa D, Ohmiya N, Nakamura M, et al. Outcome after enteroscopy for patients with obscure GI bleeding: diagnostic comparison between double-balloon endoscopy and videocapsule endoscopy. Gastrointest Endosc. 2009;69(4):866-74. Doi: https://doi.org/10.1016/j.gie.2008.06.008

87. Triester SL, Leighton JA, Leontiadis GI, et al. A meta-analysis of the yield of capsule endoscopy compared to other diagnostic modalities in patients with obscure gastrointestinal bleeding. Am J Gastroenterol. 2005;100(11):2407-18. Doi: https://doi.org/10.1111/j.1572-0241.2005.00274.x

88. deLeusseA,VahediK,EderyJ,etal.Capsuleendoscopyorpush enteroscopy for first-line exploration of obscure gastrointestinal bleeding? Gastroenterology. 2007; 132(3):855-62. Doi: https://doi.org/10.1053/j.gastro.2006.12.002 
89. Kaffes AJ,Siah C,KooJH.Clinical outcomes afterdoubleballoon enteroscopy in patients with obscure GI bleeding and a positive capsule endoscopy. Gastrointest Endosc. 2007;66(2):304-9. Doi: https://doi.org/10.1016/j.gie.2007.02.044

90. Fry LC, Neumann H, Jovanovic I, et al. Capsule endoscopy increases the diagnostic yield of double balloon enteroscopy in patients being investigated for obscure gastrointestinal bleeding. Arch Gastroenterohepatol. 2012;29(1):9-14.

91. Gay G, Delvaux M, Fassler I. Outcome of capsule endoscopy in determining indication and route for push-and-pull enteroscopy. Endoscopy. 2006;38(1):49-58. Doi: https://doi. org/10.1055/s-2005-921176

92. Hendel JW, Vilmann P, Jensen T. Double-balloon endoscopy: who needs it? Scand J Gastroenterol. 2008;43(3):3637. Doi: https://doi.org/10.1080/00365520701799468

93. Riccioni ME, Urgesi R, Cianci R, et al. Negative capsule endoscopy in patients with obscure gastrointestinal bleeding reliable: recurrence of bleeding on long-term follow-up. World J Gastroenterol. 2013;19(28):4520-5. Doi: https:// doi.org/10.3748/wjg.v19.i28.4520

94. Fisher L, Lee Krinsky M, Anderson MA, et al. The role of endoscopy in the management of obscure GI bleeding. Gastrointest Endosc. 2010;72(3):471-9. Doi: https://doi. org/10.1016/j.gie.2010.04.032

95. Gerson LB. Small bowel endoscopy: cost-effectiveness of the different approaches. Best Pract Res Clin Gastroenterol. 2012;26(3):325-35. Doi: https://doi.org/10.1016/j. bpg.2012.01.018

96. Tae $\mathrm{CH}$, Shim KN. Should capsule endoscopy be the first test for every obscure gastrointestinal bleeding? Clin Endosc. 2014;47(5):409-14. Doi: https://doi.org/10.5946/ ce.2014.47.5.409

97. Viazis N, Papaxoinis K, Vlachogiannakos J, et al. Is there a role for second-look capsule endoscopy in patients with obscure GI bleeding after a nondiagnostic first test? Gastrointest Endosc. 2009;69(4):850-6. Doi: https://doi. org/10.1016/j.gie.2008.05.053

98. Teshima CW, Kuipers EJ, van Zanten SV, et al. Double balloon enteroscopy and capsule endoscopy for obscure gastrointestinal bleeding: an updated meta-analysis. J Gastroenterol Hepatol. 2011;26(5):796-801. Doi: https:// doi.org/10.1111/j.1440-1746.2010.06530.x

99. May A, Färber M, Aschmoneit I, et al. Prospective multicenter trial comparing push-and-pull enteroscopy with the single- and double-balloon techniques in patients with small- bowel disorders. Am J Gastroenterol. 2010;105(3):575-81. Doi: https://doi.org/10.1038/ajg.2009.712

100.Lenz P, Domagk D. Double-vs. single-balloon vs. spiral enteroscopy. Best Pract Res Clin Gastroenterol.2012;26(3):30313. Doi: https://doi.org/10.1016/j.bpg.2012.01.021

101.Endo H, Matsuhashi N, Inamori M, et al. Rebleeding rate after interventional therapy directed by capsule endoscopy in patients with obscure gastrointestinal bleeding. BMC Gastroenterol. 2008;8:12-7. Doi: https://doi. org/10.1186/1471-230X-8-12

102.Delvaux M, Fassler I, Gay G. Clinical usefulness of the endoscopic video capsule as the initial intestinal investigation in patients with obscure digestive bleeding: validation of a diagnostic strategy based on the patient outcome after 12 months. Endoscopy. 2004;36(12):1067-73. Doi: https://doi.org/10.1055/s-2004-826034

103.Estévez E, González-Conde B, Vázquez-Iglesias JL, et al. Diagnostic yield and clinical outcomes after capsule endoscopy in 100 consecutive patients with obscure gastrointestinal bleeding. Eur J Gastroenterol Hepatol. 2006;18(18):881-8. Doi: https://doi.org/10.1097/00042737-200608000-00014

104. Macdonald J, Porter V, McNamara D. Negative capsule endoscopy in patients with obscure GI bleeding predicts low rebleeding rates. Gastrointest Endosc. 2008;68(6):1122-7. Doi: https://doi.org/10.1016/j.gie.2008.06.054

105.Gerson LB, Batenic MA, Newsom SL, et al. Long-term outcomes after double-balloon enteroscopy for obscure gastrointestinal bleeding. Clin Gastroenterol Hepatol. 2009;7(6):664-9. Doi: https://doi.org/10.1016/j.cgh.2009.01.021

106. Fujita M, Manabe N, Honda K, et al. Long-term outcome after double-balloon endoscopy in patients with obscure gastrointestinal bleeding. Digestion. 2010;82(3):173-8. Doi: https://doi.org/10.1159/000313360

107. Shinozaki S, Yano T, Sakamoto H, et al. Long-term outcomes in patients with overt obscure gastrointestinal bleeding after negative double-balloon endoscopy. Dig Dis Sci. 2015;60(12):3691-6. Doi: https://doi.org/10.1007/ s10620-015-3792-8

108. Williamson JB, Judah JR, Gaidos JK, et al. Prospective evaluation of the long-term outcomes after deep small-bowel spiral enteroscopy in patients with obscure GI bleeding. Gastrointest Endosc. 2012;76(4):771-8. Doi: https://doi. org/10.1016/j.gie.2012.05.025 